\title{
Channel Characterisation and Link Budget of MIMO Configuration in Near Field Magnetic Communication
}

\author{
Hoang Nguyen, Johnson I. Agbinya, and John Devlin
}

\begin{abstract}
Traditional radio communication has gained significantly from using multiple input and multiple output (MIMO) architecture in the system. Many wireless applications, such as wireless LAN and cellular network, have adopted this technology to improve their system performance. However, the effect of MIMO systems has not been investigated in the case of inductive near field short range communications. The purpose of this paper is to explore a new method for increasing the magnetic communication range using MIMO. Three system models including MISO, SIMO and MIMO are proposed to characterize the number of transmitters and receivers to the link. These models have helped to extend not only the range but also the communication channel in NFMIC.
\end{abstract}

Keywords-Near-field magnetic induction communication, NFMIC, MIMO inductive communication

\section{INTRODUCTION}

$\mathbf{E}$ LECTROMAGNETIC resonant coupling circuit has received much attention since the novel experiment of wireless power transfer was created by the MIT group in 2006 [1]. In this experiment, the source coil could transmit energy over the air to the load coil and lit up 60W light bulb. However, this experiment only sent energy in one direction and the system worked with single input and single output (SISO) configuration. Furthermore, the received power was limited in range and the efficiency of energy transfer was low at 40 percent. To increase the distance, several methods have been found. For the first method, the waveguide technique in [2] or relay elements for wireless power transfer in [3] use relay coil inserted in between the transmitter and receiver. So the relay coil will lead and drive the energy from the first coil to the last coil. Therefore, the transmission range is increased. However, the waveguide method depends on the proper position of the relay chains. If the relay nodes are too closed to each other they will create reflected power between them and reduce the distance. For the second method, the cooperative relay coils [4] are introduced between the transmitter to receiver. This model can solve the problem of the reflected power between the relay nodes. However, it still relies on the position arrangement of the middle coils between the transmitter and receiver. Furthermore, both methods reduced their performances by the lateral misalignment and angle change of the relay coils or middle coils within the transmitter and receiver. Thus, this paper will present the effect of angle variation between the transmitter and receiver

The authors are with the Department of Electronic Engineering La Trobe University, Melbourne, Australia (e-mails: h6nguyen@latrobe.edu.au; j.agbinya@1atrobe.edu.au; j.devlin@latrobe.edu.au). and address the configuration to adjust the power degradation of the transceivers.

The concept of wireless power transfer has recently spread out to near field magnetic induction communication (NFMIC), where the data transfer between the transmitter and receiver is within the range called "bubble" [5] and used in many applications such as underwater communication [6], [7], body area network [4] and Internet of things [8]. The use of magnetic communication has advantage over other wireless short range communication such as Zigbee, Wifi, Bluetooth and Ultra Wide Band (UWB) including secure communication, less interference and effective multiple path communication. However, the volume of received power decays exponentially with the inverse sixth power of distance [9]. In this paper, we propose another configuration to improve the range of communication by using multiple input multiple output (MIMO) technique. The system has more than one antenna of the transmitter antenna and/or receiver antenna. MIMO increases the communication channels of system by the number of transmitter or receiver. Therefore, this could improve the frequency diversity of the system. The study will introduce three models of MIMO system in NFMIC, simulate by software and evaluate by hardware.

The remaining of the paper is presented as follows. Section II introduces the fundalmental theory of magnetic communication and the link budget in SISO system. Section III derives the magnetic communication range for MIMO system. Three MIMO system models including multiple input single output (MISO), single input multiple output (SIMO) and MIMO configuration are proposed. In the fourth section, the range and the angle between the transmitter and receiver is simulated to analyze the effect of angle on the coupling coefficient and on the distance. Several numbers of transmitters and receivers are used to validate and improve the link budget. An evaluation hardware in section $\mathrm{V}$ is designed to analyse the use of MIMO configuration on the system performance.

\section{Magnetic Induction Communication Theory}

\section{A. Single Input Single Output System}

The single magnetic induction system and the RCL equivalent circuit can be presented as in Fig. 1, where the transmitter and receiver transfer energy between two coils of radius $r_{1}$ and $r_{2}$ respectively, separated by distance $x$.

The circuit acts as two inductive coupling series RCL circuits resonating at the same resonant angular frequency $\omega_{o}=\frac{1}{\sqrt{L_{1} C_{1}}}=\frac{1}{\sqrt{L_{2} C_{2}}}$ where, $L_{1}$ and $L_{2}$ are the self 


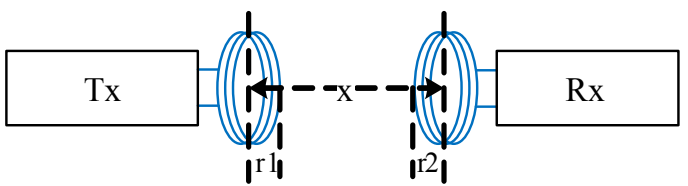

(a)

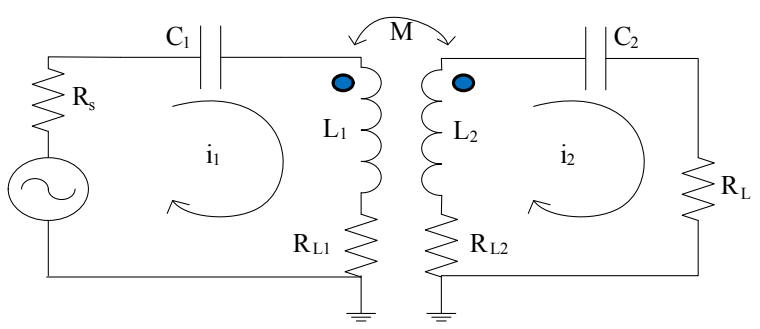

(b)

Fig. 1. NFMIC and equivalent circuit.

inductances of two coils and $C_{1}$ and $C_{2}$ are capacitors. The resistance of two coils and other losses in circuit is presented by $R_{L 1}$ and $R_{L 2}$. The source and the load impedances of the circuit are designated $R_{s}$ and $R_{L}$ respectively. Based on the Kirchoff's voltage law, the AC source voltage across the transmitting and receiving circuit are expressed as the following equation:

$$
\begin{aligned}
& \left(R_{s}+R_{L 1}+j \omega L_{1}+\frac{1}{j \omega C_{1}}\right) I_{1}+j \omega M I_{2}=V_{s} \\
& j \omega M I_{1}+\left(R_{L}+R_{L 2}+j \omega L_{2}+\frac{1}{j \omega C_{2}}\right) I_{2}=0
\end{aligned}
$$

After simplifying (1), the received power at the resonant frequency of the circuit can be derived as [9], [10], [11]:

$$
P_{R}=P_{T} Q_{T} Q_{R} \eta_{T} \eta_{R} k^{2}(x)
$$

where, $P_{T}$ and $P_{R}$ are the power at the source of the transmitter and at the load of receiver. The efficiency and the quality factor of the transmitter and the receiver are defined as:

$$
\begin{gathered}
\eta_{T}=\frac{R_{S}}{R_{L 1+R_{S}}}, \eta_{R}=\frac{R_{L}}{R_{L 2}+R_{L}} \\
Q_{T}=\frac{\omega_{o} L_{1}}{R_{L 1+R_{S}}}, Q_{R}=\frac{\omega_{o} L_{2}}{R_{L 2}+R_{L}}
\end{gathered}
$$

Equation (2) shows that the received power is proportional to the quality factor, the efficiency of the transmitter and receiver and squared power of the coupling coefficient $k$ between the transmitter and receiver. If the efficiency of the circuit is fixed, the quality factor $\mathrm{Q}$ is high, the key to get more power transmission is to increase coupling factor $k^{2}$. The coupling coefficient $k$ shows how strong energy is transferred between the coils. This is described in the equation:

$$
k=\frac{M}{\sqrt{L_{1} L_{2}}}
$$

where, $\mathrm{M}$ is the mutual inductance coupling between the transmitter and receiver. Since $k$ defines the relationship of magnetic flux linkages between two coils, $k$ can never be greater than 1. A value of $k$ measures the strength of the fluxes produced by the transmitters which are transferred to the receiver, and vice versa. Moreover, in the circuit the magnetic fluxes are created by the current density feeding into the number of turns in the coil and the interaction between the two coils. Therefore, the coupling coefficient depends on the physical dimensions of the coil. To obtain the optimal coupling between the transmitter and receiver, we can change the physical dimensions and the spatial relationship of the coils. For two single layer circular coils in Fig. 1a, the coupling coefficient can be defined by a function of the coil radius of the transmitter and the receiver $\left(r_{1}\right.$ and $\left.r_{2}\right)$ and the distance $(x)$ between them [10].

$$
k^{2}(x)=\frac{r_{1}^{3} r_{2}^{3} \pi^{2}}{\left(x^{2}+r_{1}^{2}\right)^{3}} \quad r_{2} \ll r_{1}
$$

Insert (6) into (2), the power at load of circuit can be rewritten as:

$$
P_{R}=P_{T} Q_{T} Q_{R} \eta_{T} \eta_{R} \frac{r_{1}^{3} r_{2}^{3} \pi^{2}}{\left(x^{2}+r_{1}^{2}\right)^{3}}
$$

From (7) and let $Q=Q_{T}=Q_{R}(d B), \quad \eta=\eta_{T}+$ $\eta_{R}(d B)$ and $P=P_{T}-P_{R}(d B)$, the link budget equation of SISO system can be expressed as [12], [13]:

$$
x=10^{\frac{P+Q+\eta+20 \log \pi+30 \log \left(r_{1} r_{2}\right)}{60}} 10^{10 \log \left\{\frac{1}{\left(1+\frac{r_{1}^{2}}{x^{2}}\right)^{3}}\right\}}
$$

The factor of 60 above comes from the fact that in the near field communication, the transmitted power reduces quickly, therefore severely limits the range as the distance increases between the transmitter and receiver. Consequently, NFMIC has less interference with other sources outside the effective communication range [14].

\section{B. MIMO System in NFMIC}

In RF communication, the array of channel transmission using multiple antennas in the transmitter and receiver is known as MIMO system. The multiple path fading channels could be achieved from line of sight or no line of sight. MIMO can offer a significant impact over the traditional SISO system [15]. Many researchers have studied MIMO in RF communication; however this concept has not been investigated in NFMIC. Although, the link budget equation has been presented in [16] for SISO system, it was not established in MIMO model. Therefore, this paper introduces the link budget for three system models of NFMIC including MISO, SIMO and MIMO system. These models improve the communication range and power diversity of antenna. The mathematical equation of the impact of MIMO system on the range communication between the transmitter and receiver has been derived.

MISO system: The system consists of multiple antennas at the transmitter and a single antenna at the receiver. The transmitter is assumed to use a multiple transmission coupling 


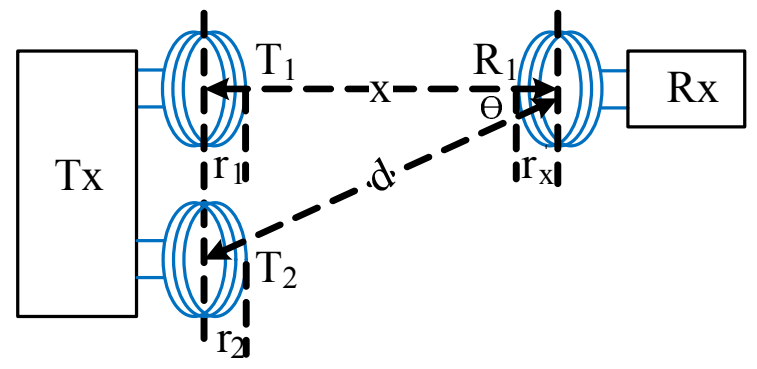

Fig. 2. MISO system.

to the receiver. In this example, we use two transmitters and one receiver. The two transmitters have coil radii of $r_{1}$ and $r_{2}$, respectively and the receiver has a coil radius of $r_{r}$. The transmitter 1 aligns with the receiver in the distance of $x$. The distance between the transmitter 2 and the receiver is $d$. The angle between $x$ and $d$ is $\theta$. Figure 2 shows the MISO system model.

At resonance, each transmitter is coupled with the receiver and the power transfer at the receiver is given by the following equation:

$$
\begin{aligned}
& P_{R 1}=P_{T} Q_{T} Q_{1} \eta_{T} \eta_{1} k_{1}^{2}(x) \\
& P_{R 2}=P_{T} Q_{T} Q_{2} \eta_{T} \eta_{2} k_{2}^{2}(x)
\end{aligned}
$$

When the radius of received antenna is smaller than those of the transmitter antenna $r_{r} \ll r_{1}, r_{2}$ the coupling efficiency between the transmitter and receiver is expressed by the following equations:

$$
\begin{aligned}
& k_{1}^{2}(x)=\frac{r_{1}^{3} r_{r}^{3}}{\left(x^{2}+r_{1}^{2}\right)^{3}} \\
& k_{2}^{2}(x)=\frac{r_{2}^{3} r_{r}^{3}}{\left((x / \cos \theta)^{2}+r_{2}^{2}\right)^{3}}
\end{aligned}
$$

The magnetic fluxes between the transmitters create interference in the system which reduces the power transfer to the receiver. This paper does not discuss the power reduction by crosstalk in the system. We assume that the transmitters are far enough from each other so the effect of crosstalk between the transmitters to the transmission is neglected. As a result, the received power in system is summarized as:

$$
P_{R}=P_{R 1}+P_{R 2}=P_{T} Q_{T} \eta_{R}\left(Q_{1} \eta_{1} k_{1}^{2}(x)+Q_{2} \eta_{2} k_{2}^{2}(x)\right)
$$

When the circuit at the transmitter and receiver is considered to be identical as in the SISO system, the quality factor and the link efficiency are unity in both circuits $Q_{1}=Q_{2}=Q_{T}$ and $\eta_{1}=\eta_{2}=\eta_{T}$. The total received power at resonant frequency is derived as:

$$
P_{R}=P_{R 1}+P_{R 2}=P_{T} Q_{T} Q_{R} \eta_{T} \eta_{R}\left(k_{1}^{2}(x)+k_{2}^{2}(x)\right)
$$

When the system is extended to $\mathrm{N}$ transmitters, the total power at the load of receiver is enhanced according to the following equation:

$$
\begin{aligned}
P_{R} & =P_{R 1}+P_{R 2}+\cdots+P_{N} \\
& =P_{T} Q_{T} Q_{R} \eta_{T} \eta_{R}\left(k_{1}^{2}(x)+k_{2}^{2}(x)+\cdots \cdots+k_{N}^{2}(x)\right)
\end{aligned}
$$

Substituting (10) into (13) and the radius of transmitters are the same $r_{1}=r_{2}=\cdots=r_{N}=r_{T}$, the received power is expressed as:

$$
P_{r}=P_{T} Q_{T} Q_{R} \eta_{T} \eta_{R} \pi^{2} r_{T}^{3} r_{R}^{3}\left(\begin{array}{c}
\frac{1}{\left(1+\frac{r_{T}^{2}}{x^{2}}\right)^{3} x^{6}}+\cdots \\
\cdots+\frac{\cos ^{6} \theta_{N}}{\left(1+\frac{r_{T}^{2} \cos ^{2} \theta_{N}}{x^{2}}\right)^{3} x^{6}}
\end{array}\right)
$$

From (8), we can see that the SISO system happens when the angle $\theta$ equals 0 degree. It means the transmitter is aligned with the receiver. If the antenna coil of transmitter and the receiver is not in the same line, the link budget equation can be derived as:

$$
x=10^{\frac{P+Q+\eta+20 \log \pi+30 \log \left(r_{T} r_{R}\right)}{60}} 10^{10 \log \left\{\frac{\cos ^{6} \theta}{\left(1+\frac{r_{T}^{2} \cos ^{2} \theta}{x^{2}}\right)^{3} x^{6}}\right\}}
$$

This equation still holds when the transmitter coil is misaligned with the received coil. It can be separated to simplify its terms as:

$$
x=x^{\prime} * \triangle x
$$

where,

$$
\begin{aligned}
x^{\prime} & =10^{\frac{P+Q+\eta+20 \log \pi+30 \log \left(r_{T} r_{R}\right)}{60}} \\
\triangle x & =10 \frac{\operatorname{lolog}\left\{\frac{\cos ^{6} \theta}{\left(1+\frac{r_{T}^{2} \cos ^{2} \theta}{x^{\prime 2}}\right)^{3} x^{6}}\right\}}{60}
\end{aligned}
$$

The first term in (16) is the link budget for SISO system when both the transmitter and receiver are aligned and the second term is the change due to the angle factor in the link budget. Replacing (14) by (16), the link budget of the MISO model in terms of angle $(\theta)$ and number of transmitters $\left(n_{T}\right)$ are given as:

$$
\begin{aligned}
& x=x^{\prime} * 10^{\left\{\sum_{\theta=0^{\circ}}^{\theta=90^{\circ}} \operatorname{lolog}\left(\frac{\cos ^{6} \theta}{\left(1+\frac{r_{T}^{2} \cos ^{2} \theta}{x^{\prime 2}}\right)^{3} x^{6}}\right)\right\}} \quad \text { if } \theta \text { diffirent } \\
& x=x^{\prime} * 10 \frac{\left\{\sum_{N=1}^{N=n_{N} T_{10 l o g}}\left(\frac{\cos ^{6} \theta}{\left(1+\frac{r_{T}^{2} \cos ^{2} \theta}{x^{\prime 2}}\right)^{3} x^{6}}\right)\right\}}{60} \text { if } \theta \text { the same } \\
& x=x^{\prime} \sum_{\theta=0^{\circ} \mid N=1}^{\theta=90^{\circ} \mid N=n_{T}} \Delta x
\end{aligned}
$$


SIMO system: the system consists of a single antenna at the transmitter and multiple antennas at the receiver. In this example, we use one transmitter and two receivers. All other parameters are the same as those of the MISO system.

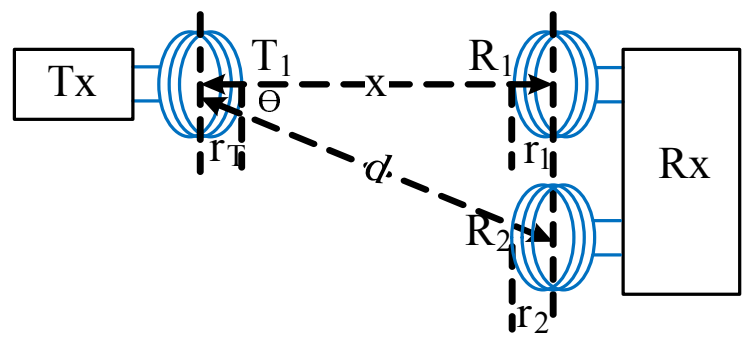

Fig. 3. NFMI SIMO system.

Similar to MISO system, at resonance, we assume that the transmitter can couple energy to all the receivers. Each receiver picks up their own energy from the transmitter. The received power at the load of each receiver is given by the following equations:

$$
\begin{aligned}
& P_{R 1}=P_{T} Q_{T} Q_{1} \eta_{T} \eta_{1} k_{1}^{2}(x) \\
& P_{R 2}=P_{T} Q_{T} Q_{2} \eta_{T} \eta_{2} k_{2}^{2}(x)
\end{aligned}
$$

Therefore, the total power of the system at resonant frequency is calculated as:

$$
\begin{aligned}
P_{r} & =P_{R 1}+P_{R 2} \\
& =P_{T} Q_{T} \eta_{T} \eta_{R}\left(Q_{1} \eta_{1} k_{1}^{2}(x)+Q_{2} \eta_{2} k_{2}^{2}(x)\right)
\end{aligned}
$$

In the case, all the receivers are identical, the quality factor and the efficiency are the same, and the received power of system at resonant frequency is rewritten as:

$$
P_{r}=P_{R 1}+P_{R 2}=P_{T} Q_{T} Q_{R} \eta_{T} \eta_{R}\left(k_{1}^{2}(x)+k_{2}^{2}(x)\right)
$$

When we have $\mathrm{N}$ receivers, the total received power is derived as:

$$
\begin{aligned}
P_{R} & =P_{R 1}+P_{R 2}+\cdots+P_{N} \\
& =P_{T} Q_{T} Q_{R} \eta_{T} \eta_{R}\left(k_{1}^{2}(x)+k_{2}^{2}(x)+\cdots+k_{N}^{2}(x)\right)
\end{aligned}
$$

We can see that equation (21) is the same as (13). Therefore, it is possible to create the same system using both MISO and SIMO configuration. However, the channel characterisation in MISO and SIMO will be different since the transmission in MISO is separated from transmitters and transmission in SIMO is separated from receivers. The difference will be shown later in section IV. Inserting (16) in (21), after deriving the equation, we have the link budget equation of the SIMO system based on angle $(\theta)$ and number of receiver $\left(n_{R}\right)$.

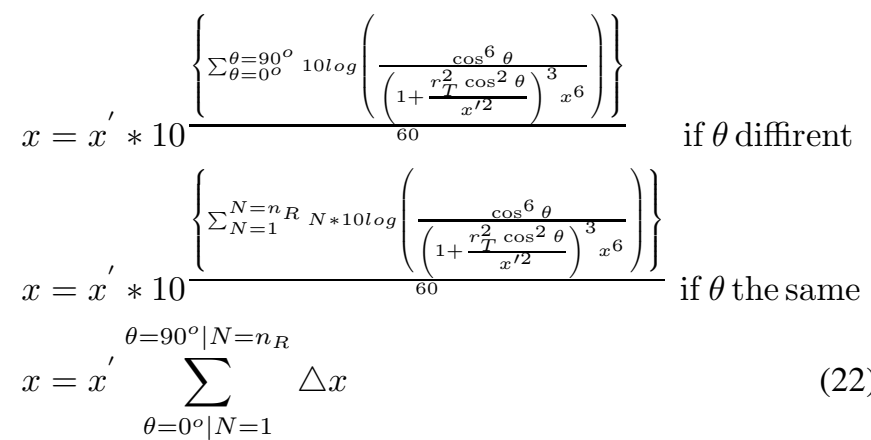

MIMO system: the system consists of multiple antennas at the transmitter and multiple antennas at the receiver. The circuit is described in Fig. 4. All other parameters are the same as those of the MISO and SIMO system. The receivers can receive separately all the signals from individual transmitter and the crosstalk between the transmitters and receivers is small. The circuits are identical to the SISO system.

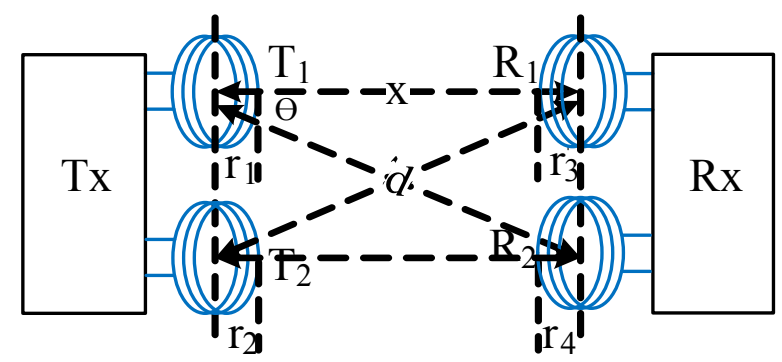

Fig. 4. MIMO system.

By combining the received power at each load of the circuit, the total received power at resonant frequency is given as:

$$
\begin{aligned}
P_{R} & =P_{R 1}+P_{R 2}+P_{R 3}+P_{R 4} \\
& =P_{T} Q_{T} Q_{R} \eta_{T} \eta_{R}\left(k_{1}^{2}(x)+k_{2}^{2}(x)+k_{3}^{2}(x)+k_{4}^{2}(x)\right)
\end{aligned}
$$

Where, $k_{1}$ and $k_{2}$ are the coupling coefficients between transmitter 1 to the receivers and $k_{3}$ and $k_{4}$ are the coupling coefficients between transmitter 2 to the receivers. After applying the coupling coefficient and substituting (15) to (23) and the radius of transmitters and receivers are the same $r_{T}=r_{R}=r$, the link budget equation of the MIMO system is yielded as:

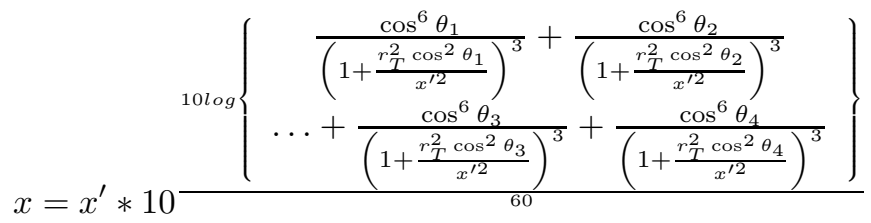

Simplifying (24) by angle $(\theta)$ and number of transmitters $\left(n_{T}\right)$ and receivers $\left(n_{R}\right)$, the link budget is given by the following expressions: 
$x=x^{\prime} * 10^{\left\{\sum_{\theta=0^{\circ}}^{\theta=90^{\circ}} \operatorname{lolog}\left(\frac{\cos ^{6} \theta}{\left(1+\frac{r^{2} \cos ^{2} \theta}{x^{\prime 2}}\right)^{3} x^{6}}\right)\right\}}$

if $\theta$ diffirent

$x=x^{\prime} * 10^{\left\{\sum_{N=1}^{N=n_{T}+n_{R}} N * 10 \log \left(\frac{\cos ^{6} \theta}{\left(1+\frac{r^{2} \cos ^{2} \theta}{x^{\prime 2}}\right)^{3} x^{6}}\right)\right\}}$. if $\theta$ the same

$x=x^{\prime} \sum_{\theta=0^{\circ} \mid N=1}^{\theta=90^{\circ} \mid N=n_{T}+n_{R}} \triangle x$

Equation (25) determines the range in NFMIC under MIMO configuration. This illustrates the impact of the number of transmitters and receivers and the angle between the transmitters and receivers to the distance.

\section{Model VALidAtion AND IMPROVEMENTS}

The simulation firstly generates the effect of the angle between the transmitter and receiver to the distance between them. The transmitter and receiver create mutual coupling in the SISO system. At the resonant frequency, the transmitter sends a signal to receiver at $25 \mathrm{dBm}$ while it moves around the receiver. The receiver detects the signal and achieves data transfer at $23 \mathrm{dBm}$. The communication range between the transmitter and receiver is measured by (8). The quality factor and the efficiency of the transmitter and receiver are $25 \mathrm{~dB}$ and 70 percent. The coil radii of the transmitter and receiver are $6 \mathrm{~cm}$ and $1.5 \mathrm{~cm}$ respectively. The increment of the angle of the transmitter used in this configuration is in the steps of 10 degree.

The result has shown that the link budget depends on the angle $\theta$ between the transmitter and receiver in Fig. 5. It can be seen that the distance between the transmitter and receiver moves up and down due to the angle change of the transmitter and receiver. The distance will be maximized at 0 degree and 180 degrees and minimized at 90 degree and 270 degree. It changes direction after each 90 degree interval. Consequently, when the transmitter and receiver are in the parallel positions, the received signal is strongest and the communication distance is maximized.

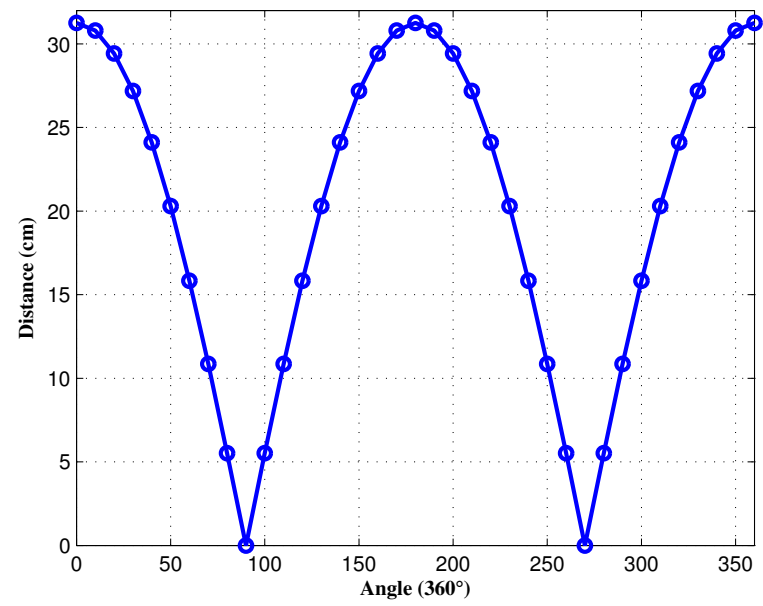

Fig. 5. Range versus the angle between transmitter and receiver.

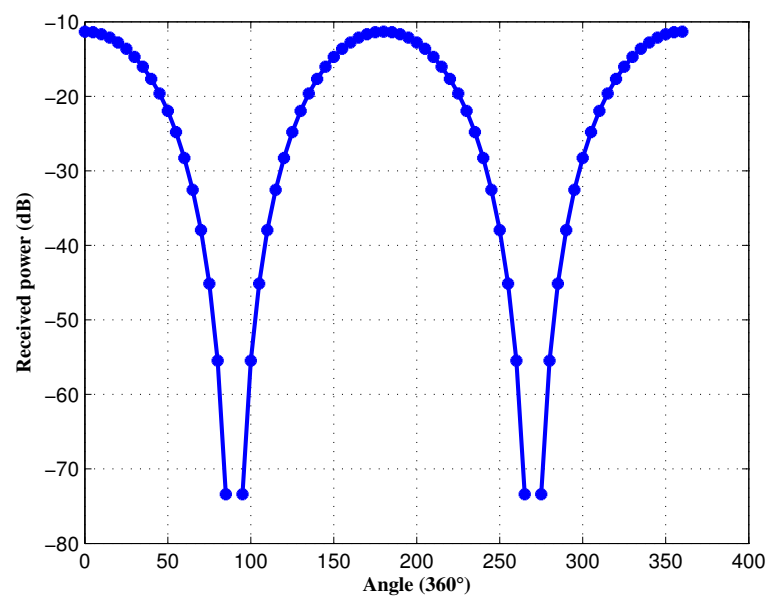

Fig. 6. Received power at load versus the angle between the coils.

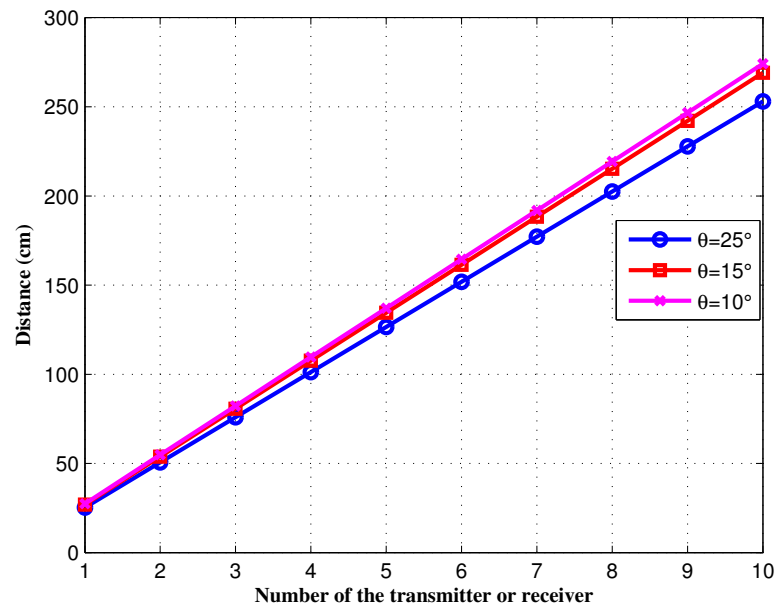

(a)

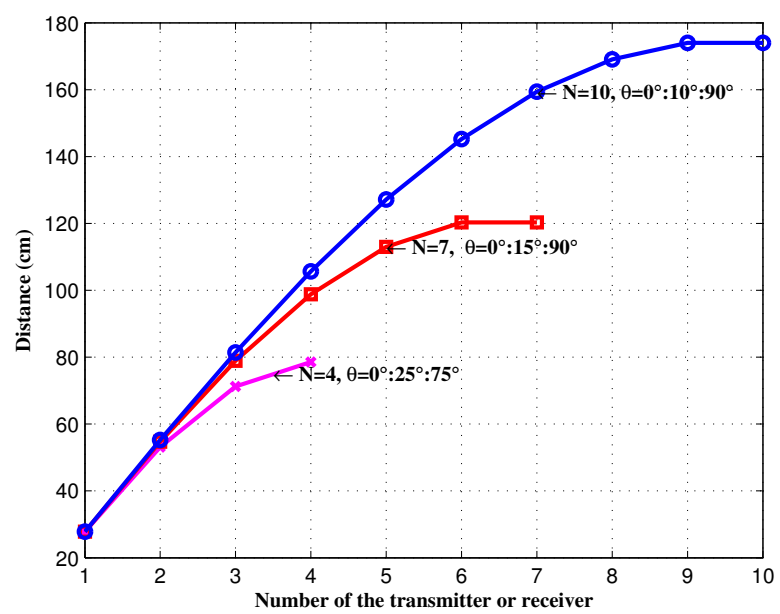

(b)

Fig. 7. NFMIC range for MISO and SIMO: a) is the same angle, b) is diffirent angle. 
When the distance between the coils is fixed at $6 \mathrm{~cm}$, the power at the receiver load is reduced quickly with the angle change between the transmitter and receiver. Figure 6 shows that the received power drops 2.5 times of the power when the angle changes from 0 degree to 45 degree and dramatically decreases after that.

Secondly, to simulate the effect of MIMO to the range extension, two situations have been exemplified. First consider the cases in which the number of transmitters and receivers is increased while the angles stay the same at 10, 15 and 25 degrees. Second, in the example it is assumed that there are four, seven and ten transmitters in the MISO system and the same numbers at the receiver in the SIMO system. The position of transmitters or receivers is separated by 25 degrees, 15 degrees and 10 degrees in the case of four, seven and ten transmitters or receivers, respectively. All other parameters are taken from the SISO system.

The analysis of the range according to the number of transmitters and receivers is shown in Fig. 7. The simulation runs for the angle theta of ten, fifteen and twenty five degrees in Fig. 7a and for four, seven and ten transmitters or receivers in Fig. 7b, indicated by dot, square and round lines, respectively. It is observed that by increasing the number of the transmitters or receivers, the range increases significantly. The range reaches $79 \mathrm{~cm}, 120 \mathrm{~cm}$ and $174 \mathrm{~cm}$ in Fig. $7 \mathrm{~b}$ as the number of the transmitters or receivers is four, seven and ten, respectively.

\section{EXPERIMENTAL EVALUATION}

SIMO: The system of one transmitter and two receivers has been demonstrated to investigate the effect of SIMO configuration to the received power. The antenna coil of the transmitter is designed as a flat spiral shape to create uniform magnetic flux to all the receivers. The inner and outer radii of the spiral coil are $2.1 \mathrm{~cm}$ and $6.1 \mathrm{~cm}$, respectively. The spiral is wound to 15 turns with a space between the turns of $2.5 \mathrm{~mm}$. The self inductance of the transmitting coil is calculated to be approximately $19.3 \mu \mathrm{H}$. The receivers are designed as single layer circular air cored coils. The received coils have radii of $2.1 \mathrm{~cm}$ and the diameter of the winding wire is $0.8 \mathrm{~mm}$. The number of turns for each coil is 11 , which equals to $7.3 \mu \mathrm{H}$ inductances. The transmitter coil is connected to $3.7 \mathrm{nF}$ capacitor and each received coil is connected to $10 \mathrm{nF}$ capacitor. Theoretically, the transmitter and receiver circuit is resonant at $590 \mathrm{KHz}$ frequency. The measurements are analyzed by the signal generator and oscilloscope. The CFG250 function generator runs the frequency at $590 \mathrm{KHz}$ and connects to the transmitter. The channels in the oscilloscope are connected to the receivers. Figure 8 shows the circuit set up and the signals at oscilloscope.

It can be seen from Fig. 8 that the transmitter is at the bottom of the panel and two receivers are at the top of the panel. The gap between the transmitter coil and the receiver 1 is called $\mathrm{d} 1$ and that between the transmitter coil and the receiver 2 is called $\mathrm{d} 2$. Both of gaps are changed randomly within $4 \mathrm{~cm}$. As the result, the output waveform of the receiver can be easily plotted and shown in Fig. 9, where channel 1 (yellow signal) and channel 2 (blue signal) are the receiver 1

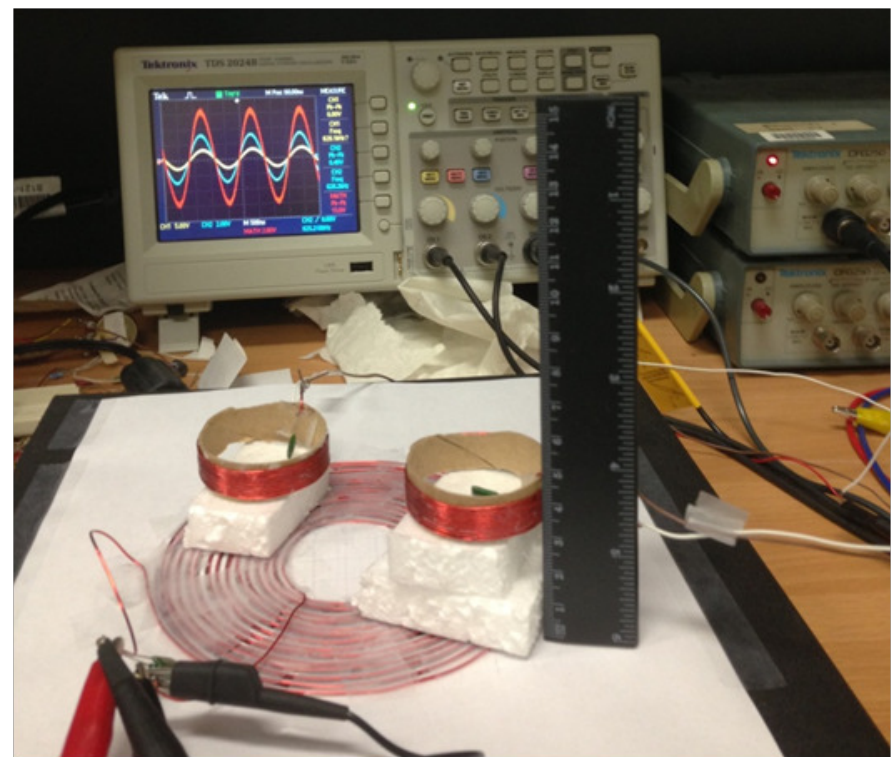

Fig. 8. SIMO system set up.

and receiver 2, respectively. The sum of two receivers is illustrated in channel 3 (red signal). It can be observed that the resonant frequency read is $621 \mathrm{KHz}$.

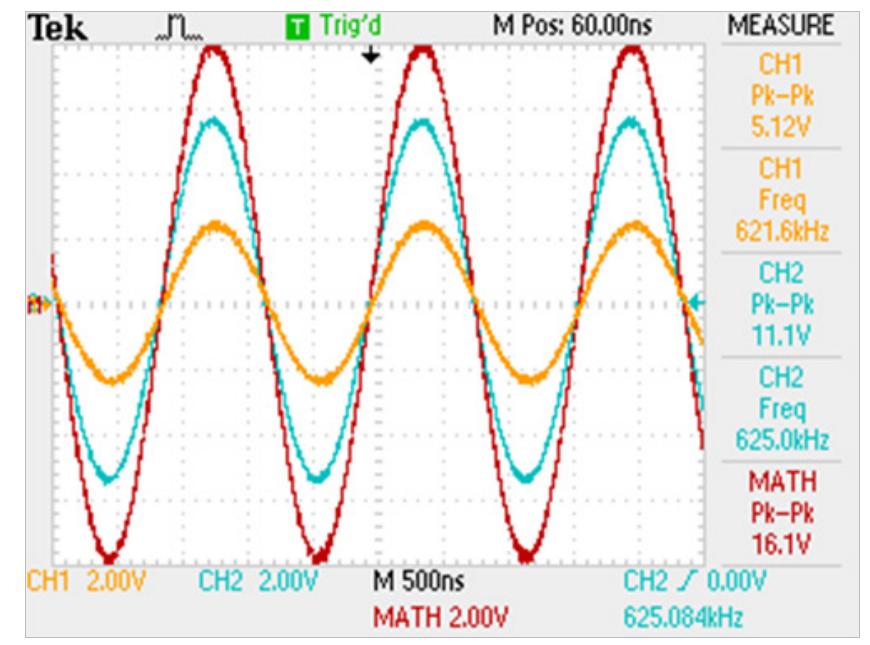

Fig. 9. Output waveform of receiver in SIMO system.

TABLE I

RECEIVED Voltage AT DIFFIRENT GAPS IN SIMO SYSTEM

\begin{tabular}{|c|c|c|c|c|}
\hline $\mathrm{d} 1(\mathrm{~cm})$ & $\mathrm{d} 2(\mathrm{~cm})$ & $\mathrm{V} 1(\mathrm{~V})$ & $\mathrm{V} 2(\mathrm{~V})$ & $\mathrm{V}$ total $(\mathrm{V})$ \\
\hline \hline 2 & 2 & 6.96 & 7.04 & 13.8 \\
\hline 2 & 3.5 & 6 & 6.48 & 12.2 \\
\hline 3.5 & 2 & 3.44 & 9.28 & 12.8 \\
\hline 3.5 & 2 & 3.6 & 12.3 & 15.7 \\
\hline 3.5 & 1.5 & 3.68 & 13.3 & 16.9 \\
\hline
\end{tabular}

It can also be seen clearly that the transmitter transferred the energy to the two receivers. Furthermore, when the distance between the transmitter and each receiver changes the voltage value at the load of each receiver is also varied. At large distances, the signal received is small and when the distance 
is small, the signal received is strong. This is because the coupling coefficient between the transmitter coil and receiver coil belongs to the physical dimension of the coils and the distance between them. The details of the output voltage at the receivers according to the distance change are introduced in Tab. I. The practical lesson shown is that it is very difficult to get the same value of received voltage when the gap is fixed since the cross coupling between two receivers impacts the magnetic flux from the transmitter to receivers. For example, when distance $\mathrm{d} 1=2 \mathrm{~cm}$ and $\mathrm{d} 2=3.5 \mathrm{~cm}$, the voltage at the receiver 1 , receiver 2 and total voltage are $6 \mathrm{~V}, 6.48 \mathrm{~V}$ and $12.2 \mathrm{~V}$, respectively. But when distances $\mathrm{d} 1=3.5 \mathrm{~cm}$ and $\mathrm{d} 2=2 \mathrm{~cm}$, the voltage at the receiver 1 , receiver 2 and total voltage are different at $3.44 \mathrm{~V}, 9.28 \mathrm{~V}$ and $12.8 \mathrm{~V}$, respectively.

MISO: The system of two transmitters and one receiver is built to demonstrate the MISO. The design is swapped between the coils of the transmitter and receiver in SIMO system. The two singular coils are the transmitters, which are connected to the sinusoidal wave of the signal generator. The spiral coil acts as the receiver. Two transmitters and receivers are also linked to channel 1, channel 2 and channel 3, respectively and are measured with the oscilloscope. Three capacitors $3.7 \mathrm{nF}$ and $2 \times 10 \mathrm{nF}$ is also added in parallel to the receiver and transmitters. Consequently, the voltage of transmitters and receiver is illustrated in Fig. 10. We can see that the circuit is resonant at $662.3 \mathrm{KHz}$ frequency and the receiver voltage shows a phase shift. The transmitter signals are two upper waveforms (yellow and blue signals) and have a magnitude of equal value $15 \mathrm{~V}$. The received signal is presented in channel 3 (purple colour) and has a peak to peak voltage of $672 \mathrm{mV}$.

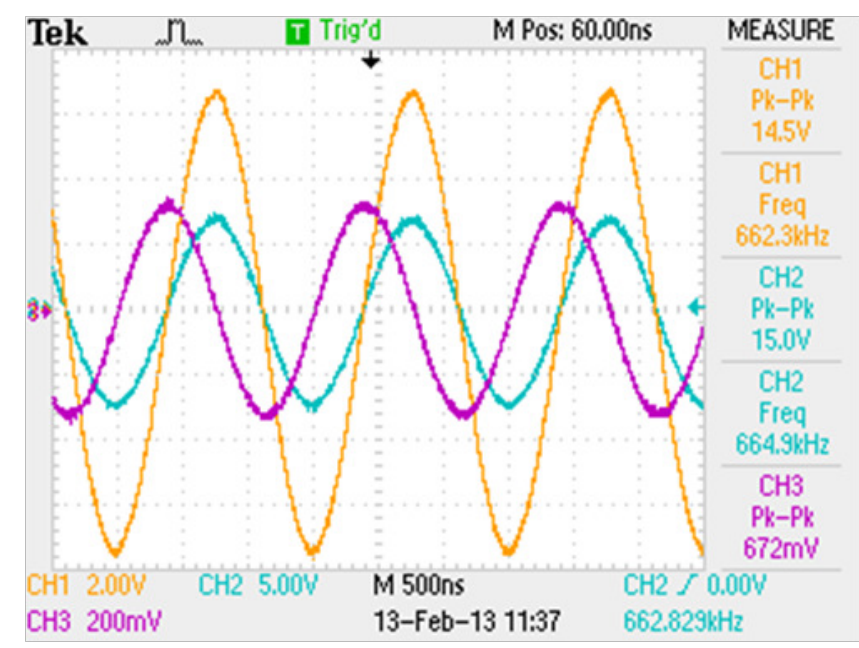

Fig. 10. The output voltage of receiver and transmitter in MISO system.

It has shown that the output signal of the receiver did not get much energy from the transmitters since the system is transferred from the low quality factor $\mathrm{Q}$ of transmitter to the high quality factor $\mathrm{Q}$ of the receiver. Using (4), the quality factor of the transmitter and receiver equal to 30 and 80 , respectively. The received voltages combine all the signals from the transmitters into one at the receiver. Because it cannot separate the channel of the received signal from each transmitter. The detail of voltage responded due to coupling
TABLE II

RECIEVEd Voltage AT DifFIRENT POSITION IN MISO SYSTEM

\begin{tabular}{|c|c|c|}
\hline $\mathrm{d} 1(\mathrm{~cm})$ & $\mathrm{d} 2(\mathrm{~cm})$ & Received voltage $(\mathrm{mV})$ \\
\hline \hline 2 & 2 & 672 \\
\hline 2 & 3.5 & 600 \\
\hline 4 & 2 & 632 \\
\hline 4 & 3.5 & 568 \\
\hline
\end{tabular}

coefficient is reported in Tab. II.

\section{CONCLUSiON}

The paper has detailed a link budget for MIMO configuration in NFMIC. The impact of angle to the distance and the power loss between the transmitter and receiver is observed. To improve the communication and the range in NFMIC, the new method based on MIMO configuration is used and validated by simulation. An example of communication channel in models of MISO, SIMO and MIMO system are introduced. This model suggests that increasing the number of transmitters is recommended for uplink communication and increasing the number of receivers is recommended for downlink communication. The experiment shows that the use of MIMO configuration can diversify the channel transmission of the system of communication.

\section{ACKNOWLEDGEMENT}

The authors would like to thank Department of Electronics Engineering, La Trobe University for providing the financial support for this project.

\section{REFERENCES}

[1] A. Kurs, A. Karalis, R. Moffatt, J. D. Joannopoulos, P. Fisher, and M. Soljacic, "Wireless Power Transfer via Strongly Coupled Magnetic Resonances," Science express, vol. 317, pp. 83-86, 6 July 2007.

[2] Z. Sun and I. F. Akyildiz, "Magnetic Induction Communications for Wireless Underground Sensor Networks," IEEE Transactions on Antennas and Propagation, vol. 58, pp. 2426-2435, 2010.

[3] M. Dionigi and M. Mongiardo, "Magnetically coupled resonant Wireless Power Transmission systems with relay elements," in Microwave Workshop Series on Innovative Wireless Power Transmission: Technologies, Systems, and Applications (IMWS), 2012 IEEE MTT-S International, 2012, pp. 223-226.

[4] M. Masihpour and J. I. Agbinya, "Cooperative relay in Near Field Magnetic Induction: A new technology for embedded medical communication systems," in Proceedings of the Fifth International Conference on Broadband and Biomedical Communications, 2010, pp. 1-6.

[5] R. Bansal, "Near-field magnetic communication," IEEE Antennas and Propagation Magazine, vol. 46, pp. 1140-115, 2004.

[6] M. C. Domingo, "Magnetic Induction for Underwater Wireless Communication Networks," IEEE Transactions on Antennas and Propagation, vol. 60, pp. 2929-2939, 2012.

[7] B. Gulbahar and O. B. Akan, "A Communication Theoretical Modeling and Analysis of Underwater Magneto-Inductive Wireless Channels," IEEE Transactions on Wireless Communications, vol. 11, pp. 33263334, 2012.

[8] J. I. Agbinya and S. Lal, "A high capacity near-field inductive coupled MISO communication system for Internet of Things," in 6th International Conference onBroadband and Biomedical Communications (IB2Com), 2011, pp. 112-117.

[9] J. I. Agbinya and M. Masihpour, "Power equations and capacity performance of Magnetic induction body area network nodes," in Proceedings of the Fifth International Conference on Broadband and Biomedical Communications, 2010, pp. 1-6.

[10] H. C. Jing and Y. E. Wang, "Capacity performance of an inductively coupled near field communication system," in Proceedings of the IEEE Antennas and Propagation Society International Symposium, 2008, pp. $1-4$. 
[11] U. Azad, H. C. Jing, and Y. E. Wang, "Link Budget and Capacity Performance of Inductively Coupled Resonant Loops," IEEE Transactions on Antennas and Propagation, vol. 60, pp. 2453-2461, 2012.

[12] J. I. Agbinya and M. Masihpour, "Near field magnetic induction communication Link Budget: Agbinya-Masihpour model," in Proceedings of the Fifth International Conference on Broadband and Biomedical Communications, 2010, pp. 1-6.

[13] J. I. Agbinya, "A magneto-inductive link budget for wireless power transfer and inductive communication systems," Progress In Electromagnetic Research C, vol. 37, pp. 15-28, 2013.
[14] H. G. Schantz, "Near field propagation law \& a novel fundamental limit to antenna gain versus size," in Proceedings of the IEEE Antennas and Propagation Society International Symposium, 2005, pp. 237-240, vol. 3A.

[15] B. Holter, "On the capacity of the MIMO channel: A tutorial introduction," in IEEE Norwegian Symppsium on Signal Processing, Trondheim, Norway, 2001, pp. 167-172.

[16] J. I. Agbinya and M. Masihpour, "Magnetic induction channel models and link budgets: A comparison between two Agbinya-Masihpour models," in Third International Conference on Communications and Electronics (ICCE), 2010, pp. 400-405. 